\title{
Fractional Variational Iteration Method and Its Application to Fractional Partial Differential Equation
}

\author{
Asma Ali Elbeleze, ${ }^{1}$ Adem Kılıçman, ${ }^{2}$ and Bachok M. Taib ${ }^{1}$ \\ ${ }^{1}$ Faculty of Science and Technology, Universiti Sains Islam Malaysia, 71800 Nilai, Malaysia \\ ${ }^{2}$ Department of Mathematics, Faculty of Science, University Putra Malaysia, 43400 UPM, Serdang, Selangor Darul Ehsan, Malaysia
}

Correspondence should be addressed to Adem Kılıçman; akilicman@putra.upm.edu.my

Received 13 March 2013; Accepted 5 June 2013

Academic Editor: Mufid Abudiab

Copyright (c) 2013 Asma Ali Elbeleze et al. This is an open access article distributed under the Creative Commons Attribution License, which permits unrestricted use, distribution, and reproduction in any medium, provided the original work is properly cited.

We use the fractional variational iteration method (FVIM) with modified Riemann-Liouville derivative to solve some equations in fluid mechanics and in financial models. The fractional derivatives are described in Riemann-Liouville sense. To show the efficiency of the considered method, some examples that include the fractional Klein-Gordon equation, fractional Burgers equation, and fractional Black-Scholes equation are investigated.

\section{Introduction}

The topic of fractional calculus (theory of integration and differentiation of an arbitrary order) was started over 300 years ago. Recently, fractional differential equations have attracted many scientists and researchers due to the tremendous use in fluid mechanics, mathematical biology, electrochemistry, and physics. For example, differential equations with fractional order have recently proved to be suitable tools to modeling of many physical phenomena [1] and the fluid-dynamic traffic model with fractional derivative [2], and nonlinear oscillation of earthquake can be modeled with fractional derivatives [3].

There are several types of time fractional differential equations.

(1) Fractional Klein-Gordon equations

$$
\begin{gathered}
\frac{\partial^{\alpha} u(x, t)}{\partial t^{\alpha}}-\frac{\partial^{2} u(x, t)}{\partial x^{2}}+a u(x, t)+b u^{2}+c u^{3} \\
=f(x, t), \quad x \in R .
\end{gathered}
$$

This model is obtained by replacing the order time derivative with the fractional derivative of order $\alpha$. The linear and nonlinear Klein-Gordon equations are used to modeling many problems in classical and quantum mechanics and condensed matter physics.
For example, nonlinear sine Klein-Gordon equation models a Josephson junction $[4,5]$.

(2) Fractional Burger's equation

$$
\frac{\partial^{\alpha} u(x, t)}{\partial t^{\alpha}}=\frac{\partial^{2} u(x, t)}{\partial x^{2}}+\frac{\partial u(x, t)}{\partial x}+f(x, t), \quad x \in R .
$$

In general, fractional Burger's model is derived from well-known Burger's equation model by replacing the ordinary time derivatives to fractional order time derivatives. Reference [6] has investigated unsteady flows of viscoelastic fluids with fractional Burger's model and fractional generalized Burger's model through channel (annulus) tube and solutions for velocity field.

(3) Fractional Black-Scholes European option pricing equations

In financial model the fractional Black-Scholes equation is obtained by replacing the order of derivative with a fractional derivative order [10].

$$
\begin{array}{r}
\frac{\partial^{\alpha} v}{\partial t^{\alpha}}+\frac{\sigma x^{2}}{2} \frac{\partial^{2} v}{\partial x^{2}}+r(t) x \frac{\partial v}{\partial x}-r(t) v=0, \\
(x, t) \in R^{+} \times(0, T), 0<\alpha \leq 1,
\end{array}
$$


where $v(x, t)$ is the European call option price at asset price $x$ and at time $t, T$ is the maturity, $r(t)$ is the riskfree interest rate, and $\sigma(x, t)$ represents the volatility function of underlying asset.

The payoff functions are

$$
\begin{aligned}
& v_{c}(x, t)=\max (x-E, 0), \\
& v_{p}(x, t)=\max (E-x, 0),
\end{aligned}
$$

where $v_{c}(x, t)$ and $v_{p}(x, t)$ are the value of the European call and put options, respectively, $E$ denotes the expiration price for the option, and the function $\max (x, 0)$ gives the large value between $x$ and 0 . The Black-Scholes equation is one of the most significant mathematical models for a financial market. This equation is used to submit a reasonable price for call or put options based on factors such as underlying stock volatility and days to expiration.

Formerly, [7] investigated approximate analytical solution of fractional nonlinear Klein-Gordon equation (1) when $0<\alpha \leq 1$ by using HPM, while [8] solved this equation by using HAM also when $1 \leq \alpha<2$. Reference [9] solved the coupled Klein-Gordon equation with time fractional derivative by ADM. References [10, 11] solved fractional Black-Scholes equations by using HPM using Sumudu and Laplace transforms, respectively. Reference [12] gave the exact solution of fractional Burgers equation, while [13] used DTM to find the approximate and exact solution of space- and time fractional Burgers equations. Reference [14] solved this equation by using VIM.

The variational iteration method [15-29] is one of approaches to provide an analytical approximation solutions to linear and nonlinear problems. The fractional variational iteration method with Riemann-Liouville derivative was proposed by $\mathrm{Wu}$ and Lee [30] and applied to solve time fractional and space fractional diffusion equations. Furthermore $\mathrm{Wu}$ [31] explained a possible use of the fractional variational iteration method as a fractal multiscale method. Recently fractional variational iteration method has been used to obtain approximate solutions of fractional Riccati differential equation [32].

The objective of this paper is to extend the application of the fractional variational iteration method to obtain analytical approximate solution for some fractional partial differential equations. These equations include fractional KleinGordon equation (1), Burgers equation (2), and fractional Black-Scholes equations (3).

Motivated and inspired by the ongoing research in this field, we will consider the following time fractional differential equation:

$$
\begin{aligned}
\frac{\partial^{\alpha} u(x, t)}{\partial t^{\alpha}} & =R[x] u(x, t)+q(x, t), \\
0 & <\alpha \leq 1, x \in \mathbf{R}, t>0,
\end{aligned}
$$

with initial condition

$$
u(x, 0)=f(x)
$$

where $\partial^{\alpha} / \partial t^{\alpha}$ is modified Riemann-Liouville derivative [3335] of order $\alpha$ defined in Section 2, $f(x)$ and $q(x, t)$ are continuous functions, $R[x] u(x, t)$ are linear and nonlinear operators, and $u(x, t)$ is unknown function.

To solve the problem (1)-(2), we consider the FVIM in this work. This method is based on variational iteration method $[19,36]$ and modified Riemann-Liouville derivatives proposed by Jumarie.

This paper is organized as follows. in Section 2 some basic definitions of fractional calculus theory are given. In Section 3, the solution procedure of the fractional iteration method is given; we present the application of the FVIM for some fractional partial differential equations in Section 4. The conclusions are drawn in Section 5.

\section{Fractional Calculus}

\subsection{Fractional Derivative via Fractional Difference}

Definition 1. The left-sides Riemann-Liouville fractional integral operator of order $\alpha \geq 0$, of a function $f \in C_{\mu}, \mu \geq-1$, is a defined as

$$
\begin{array}{r}
J^{\alpha} f(x)=\frac{1}{\Gamma(\alpha)} \int_{0}^{x}(x-t)^{\alpha-1} f(t) d t, \quad \alpha>0, \\
x>0, J^{0} f(x)=f(x) .
\end{array}
$$

Definition 2. The modified Riemann-Liouville derivative [34, 35] is defined as

$$
D_{\alpha}^{x} f(x)=\frac{1}{\Gamma(n-\alpha)} \frac{d^{n}}{d x^{n}} \int_{0}^{x}(x-t)^{n-\alpha}(f(t)-f(0)) d t
$$

where $x \in[0,1], n-1 \leq \alpha<n$, and $n \geq 1$.

Definition 3. Let $f: R \rightarrow R, x \rightarrow f(x)$ denote a continuous (but not necessarily differentiable) function, and let $h>0$ denote a constant discretization span. Define the forward operator $\mathrm{FW}(h)$ by the equality

$$
\mathrm{FW}(h) f(x):=f(x+h) .
$$

Then the fractional difference of order $\alpha, 0<\alpha<1$, of $f(x)$ is defined by the expression

$$
\begin{aligned}
\Delta^{(\alpha)} f(x) & :=(\mathrm{FW}-1)^{\alpha} f(x) \\
& =\sum_{k=0}^{\infty}(-1)^{k}\left(\begin{array}{l}
\alpha \\
k
\end{array}\right) f[x+(a-k) h],
\end{aligned}
$$

and its fractional derivative of order $\alpha$ is defined by the limit

$$
f^{\alpha}(x)=\lim _{x \rightarrow 0} \frac{\Delta^{(\alpha)}[f(x)-f(0)]}{h^{\alpha}} .
$$

Equation (11) is defined as Jumarie fractional derivative of order $\alpha$ which is equivalent to (8). For more details we refer the reader to [35]. 
For $0<\alpha \leq 1$, some properties of the fractional modified Riemann-Liouville derivative.

Fractional Leibnitz product law:

$$
{ }_{0} D_{x}^{\alpha}(u v)=u^{(\alpha)} v+u v^{(\alpha)}
$$

fractional Leibnitz formulation:

$$
{ }_{0} I_{x}^{\alpha} D_{x}^{\alpha}(u v)=f(x)-f(0)
$$

The fractional integration by parts formula:

$$
{ }_{a} I_{b}^{\alpha}\left(u^{(\alpha)} v\right)=\left.(u v)\right|_{a} ^{b}-{ }_{a} I_{b}^{\alpha}\left(u v^{(\alpha)}\right)
$$

Definition 4. Fractional derivative of compounded function $[34,35]$ is defined as

$$
d^{\alpha} f \cong \Gamma(1+\alpha) d f, \quad 0<\alpha<1
$$

Definition 5 (see $[34,35])$. The integral with respect to $(d t)^{\alpha}$ is defined as the solution of the fractional differential equation

$$
d x \cong f(x)(d t)^{\alpha}, \quad t \geq 0, x(0)=0,0<\alpha<1 .
$$

Lemma 6 (see $[34,35])$. Let $f(x)$ denote a continuous function; then the solution of (2) is defined as

$$
\begin{aligned}
y & =\int_{0}^{x} f(\tau)(d \tau)^{\alpha} \\
& =\alpha \int_{0}^{x}(x-\tau)^{\alpha-1} f(\tau) d \tau, \quad 0<\alpha<1,
\end{aligned}
$$

that is,

$$
\begin{aligned}
J^{\alpha} f(x) & =\left(\frac{1}{\Gamma(\alpha)}\right) \int_{0}^{x}(x-\tau)^{\alpha-1} f(\tau) d \tau \\
& =\frac{1}{(\Gamma(\alpha+1))} \int_{0}^{x} f(\tau)(d \tau)^{\alpha} .
\end{aligned}
$$

For example, with $f(x)=x^{\beta}$ in (7), one obtains

$$
\int_{0}^{x} t^{\beta}(d t)^{\alpha}=\frac{\Gamma(\beta+1) \Gamma(\alpha+1)}{\Gamma(\alpha+\beta+1)} x^{\alpha+\beta}, \quad 0<\alpha<1 .
$$

Definition 7. The Mittag-Leffler function $E_{\alpha}(z)$ with $\alpha>0$ is defined by the following series representation, valid in the whole complex plane [37]:

$$
E_{\alpha}(z)=\sum_{0}^{\infty} \frac{z^{n}}{\Gamma(\alpha n+1)} .
$$

\section{Fractional Variational Iteration Method}

To describe the solution procedure of fractional variational iteration method, we consider the time-fractional differential equations (1)-(3).

According to variational iteration method we construct the following correction function:

$$
\begin{aligned}
& u_{n+1}(x, t) \\
& =u_{n}(x, t)+J_{t}^{\alpha}\left[\mu\left(\frac{\partial^{\alpha} u(x, s)}{\partial s^{\alpha}}-R[x] \tilde{u}(x, s)-q(x, s)\right)\right] \\
& =u_{n}(x, t)+\frac{1}{\Gamma(\alpha)} \\
& \quad \times \int_{0}^{t}(t-s)^{\alpha-1}\left\{\mu ( s ) \left(\frac{\partial^{\alpha} u(x, s)}{\partial s^{\alpha}}\right.\right. \\
& \quad-R[x] \tilde{u}(x, s)-q(x, s))\} d s,
\end{aligned}
$$

where $\mu$ is the general Lagrange multiplier which can be defined optimally via variational theory [22] and $\tilde{u}(x, t)$ is the restricted variation, that is, $\delta \widetilde{u}(x, t)=0$.

By using (7), we obtain a new correction functional

$$
\begin{aligned}
& u_{n+1}(x, t)= u_{n}(x, t)+\frac{1}{\Gamma(\alpha+1)} \\
& \times \int_{0}^{t}\left\{\mu ( s ) \left(\frac{\partial^{\alpha} u(x, s)}{\partial s^{\alpha}}-R[x] \tilde{u}(x, s)\right.\right. \\
&-q(x, s))\}\left(d s^{\alpha}\right) .
\end{aligned}
$$

Making the above functional stationary the following conditions can be obtained:

$$
\begin{aligned}
& \delta u_{n+1}(x, t)= \delta u_{n}(x, t)+\frac{\delta}{\Gamma(\alpha+1)} \\
& \times \int_{0}^{t}\left\{\mu ( s ) \left(\frac{\partial^{\alpha} u(x, s)}{\partial s^{\alpha}}-R[x] \tilde{u}(x, s)\right.\right. \\
&-q(x, s))\}\left(d s^{\alpha}\right) .
\end{aligned}
$$

Now, we can get the coefficients of $\delta u$ to zero:

$$
1+\mu(s)=0, \quad \frac{\partial^{\alpha} \mu(s)}{\partial s^{\alpha}}=0 .
$$

So, the generalized Lagrange multiplier can be identified as

$$
\mu=-1 \text {. }
$$


Then we obtain the following iteration formula by substituting (25) in (23):

$$
\begin{aligned}
& \delta u_{n+1}(x, t)= \delta u_{n}(x, t)-\frac{\delta}{\Gamma(\alpha+1)} \\
& \times \int_{0}^{t}\left\{\mu ( s ) \left(\frac{\partial^{\alpha} u(x, s)}{\partial s^{\alpha}}-R[x] \widetilde{u}(x, s)\right.\right. \\
&-q(x, s))\}\left(d s^{\alpha}\right),
\end{aligned}
$$

where $0<\alpha \leq 1$ and $u_{0}(x, t)$ is an initial approximation which can be freely chosen if it satisfies the initial and boundary conditions of the problem.

\section{Applications}

In this section, we have applied fractional variational iteration method (FVIM) to fractional partial differential equations.

Example 8. In this example we consider the following fractional nonlinear Klein-Gordon differential equation:

$$
\frac{\partial^{\alpha} u}{\partial t^{\alpha}}-\frac{\partial^{2} u}{\partial x^{2}}+u^{2}=0, \quad t \geq 0,0<\alpha \leq 1
$$

subject to initial condition

$$
y(x, 0)=1+\sin (x)
$$

Substituting ( $a=0, b=0$ and $c=1)$ in (1). Construction the iteration formula as follows:

$$
\begin{aligned}
u_{n+1}(x, t)= & u_{n}(x, t)-\frac{1}{\Gamma(\alpha+1)} \\
& \times \int_{0}^{t}\left\{\frac{\partial^{\alpha} u_{n}}{\partial s^{\alpha}}-\frac{\partial^{2} u_{n}}{\partial x^{2}}+u_{n}^{2}\right\}(d s)^{\alpha} .
\end{aligned}
$$

Taking the initial value $u_{0}(x, t)=1+\sin (x)$ we can derive the first approximate $u_{1}(x, t)$ as follows:

$$
\begin{aligned}
u_{1}(x, t)= & u_{0}(x, t)-\frac{1}{\Gamma}(\alpha+1) \\
& \times \int_{0}^{t}\left\{\frac{\partial^{\alpha} u_{0}}{\partial s^{\alpha}}-\frac{\partial^{2} u_{0}}{\partial x^{2}}+u_{0}^{2}\right\}(d s)^{\alpha} \\
= & 1+\sin (x)-\frac{t^{\alpha+1}}{\Gamma(\alpha+1)} \\
& \times\left(1+3 \sin (x)+\sin ^{2}(x)\right),
\end{aligned}
$$

$$
\begin{aligned}
u_{2}(x, t)= & u_{1}(x, t)-\frac{1}{\Gamma(\alpha+1)} \\
& \times \int_{0}^{t}\left\{\frac{\partial^{\alpha} u_{1}}{\partial s^{\alpha}}-\frac{\partial^{2} u_{1}}{\partial x^{2}}+u_{1}^{2}\right\}(d s)^{\alpha} \\
= & 1+\sin (x)-\frac{t^{\alpha+1}}{\Gamma(\alpha+1)}\left(1+3 \sin (x)+\sin ^{2}(x)\right) \\
& +\frac{t^{2 \alpha+1}}{\Gamma(2 \alpha+1)}\left(11 \sin (x)+12 \sin ^{2}(x)+2 \sin ^{3}(x)\right),
\end{aligned}
$$

$$
\begin{aligned}
u_{3}(x, t)= & u_{2}(x, t)-\frac{1}{\Gamma(\alpha+1)} \\
& \times \int_{0}^{t}\left\{\frac{\partial^{\alpha} u_{2}}{\partial s^{\alpha}}-\frac{\partial^{2} u_{2}}{\partial x^{2}}+u_{2}^{2}\right\}(d s)^{\alpha} \\
= & 1+\sin (x)-\frac{t^{\alpha}}{\Gamma(\alpha+1)}\left(1+3 \sin (x)+\sin ^{2}(x)\right) \\
& +\frac{t^{2 \alpha}}{\Gamma(2 \alpha+1)}\left(11 \sin (x)+12 \sin ^{2}(x)+2 \sin ^{3}(x)\right) \\
& +\frac{t^{3 \alpha}}{\Gamma(3 \alpha+1)}\left(18-57 \sin (x)-160 \sin ^{2}(x)\right. \\
& \left.-82 \sin ^{3}(x)-10 \sin ^{4}(x)\right) .
\end{aligned}
$$

Thus, the approximate solution is

$$
\begin{aligned}
u(x, t)= & 1+\sin (x)-\frac{t^{\alpha}}{\Gamma(\alpha+1)} \\
& \times\left(1+3 \sin (x)+\sin ^{2}(x)\right)+\frac{t^{2 \alpha}}{\Gamma(2 \alpha+1)} \\
& \times\left(11 \sin (x)+12 \sin ^{2}(x)+2 \sin ^{3}(x)\right) \\
& +\frac{t^{3 \alpha}}{\Gamma(3 \alpha+1)}\left(18-57 \sin (x)-160 \sin ^{2}(x)\right. \\
& \left.\quad-82 \sin ^{3}(x)-10 \sin ^{4}(x)\right)+\cdots
\end{aligned}
$$

In Figures 1 and 2 we have shown the surface of $u(x, t)$ corresponding to the values $\alpha=0.01,0.5,1$ for FVIM and HPM; the two figures indicate that the differences among VIM and HPM, and the exact solution in Example 8 are negligible when $\alpha=0.5,1$ while when $\alpha=0.01$ the results of VIM and HPM somewhat diverge from the exact solution. 


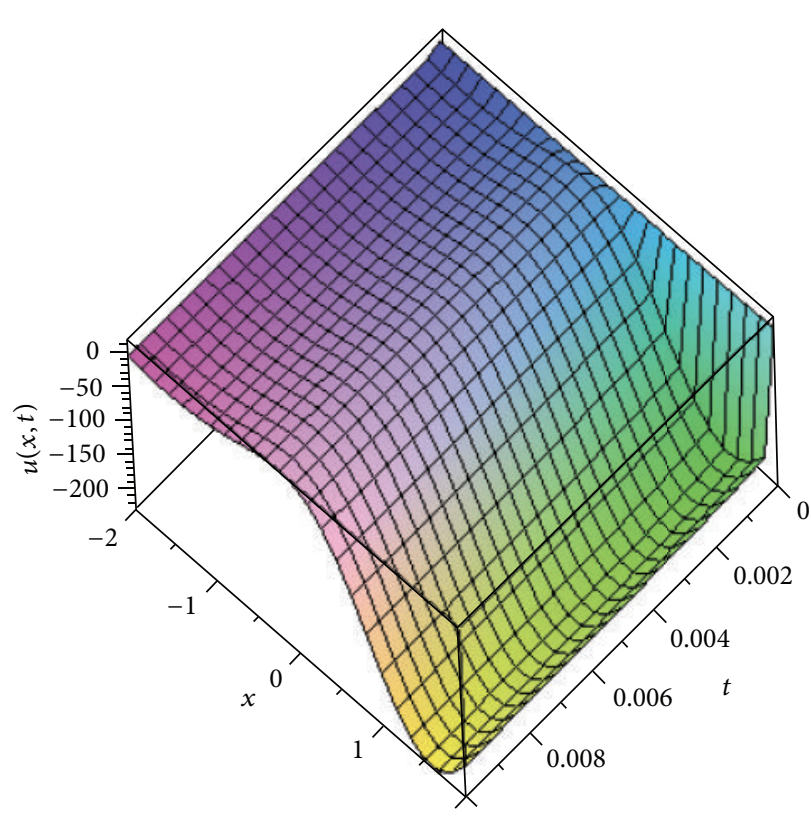

(a)

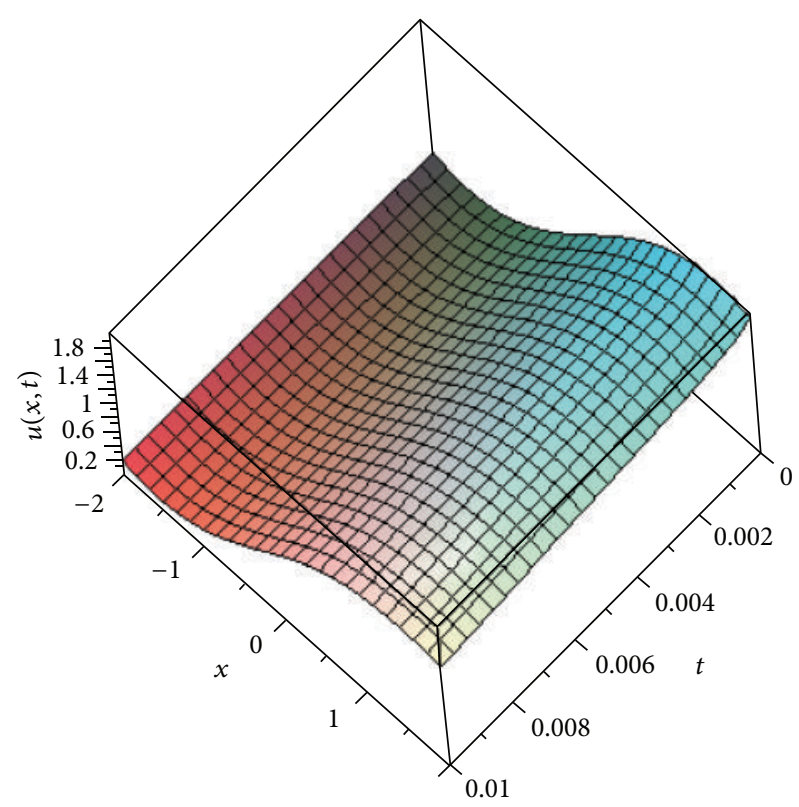

(c)

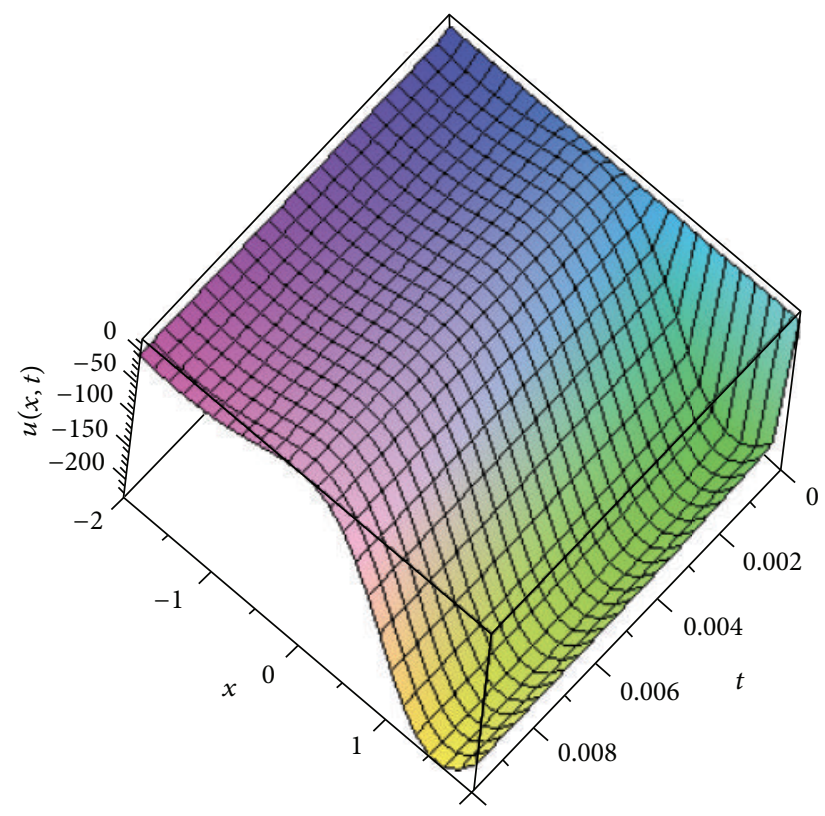

(b)

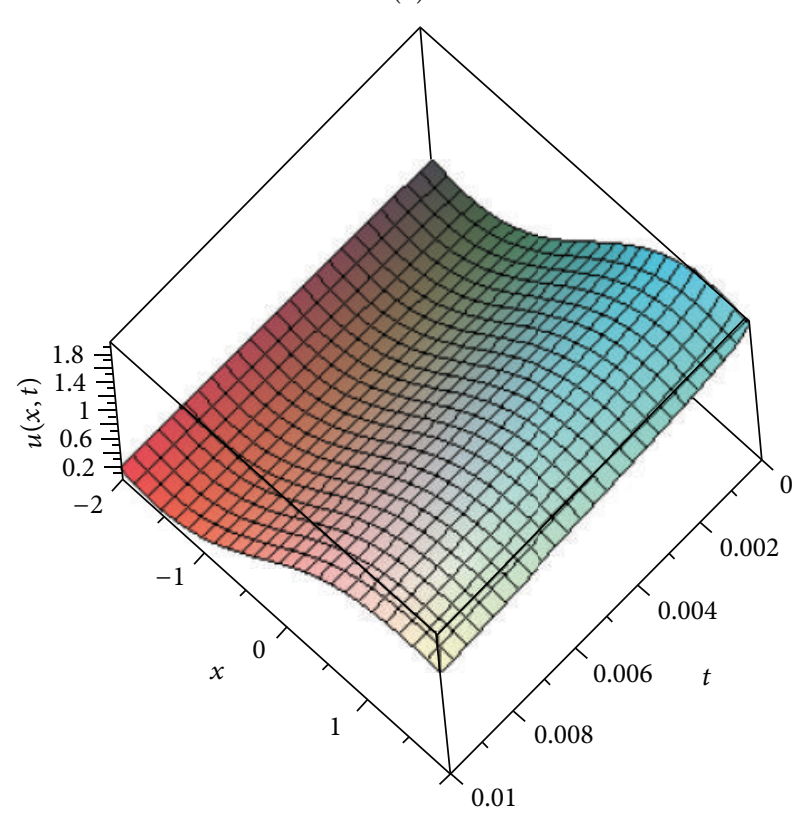

(d)

FIGURE 1: The surface shows the solution $u(x, t)$ for (27) with initial condition (28): FVIM results are, respectively, (a) $\alpha=0.01$ and (c) $\alpha=0.5$; HPM [7] results are, respectively, (b) $\alpha=0.01$ and (d) $\alpha=0.5$.

Example 9. We consider the one-dimensional linear inhomogeneous fractional Burger equation

$$
\begin{array}{r}
\frac{\partial^{\alpha} u}{\partial t^{\alpha}}+\frac{\partial u}{\partial x}-\frac{\partial^{2} u}{\partial x^{2}}=\frac{2 t^{2-\alpha}}{\Gamma(3-\alpha)}+2 x-2, \\
t>0, x \in R, 0<\alpha \leq 1,
\end{array}
$$

subject to initial condition

$$
u(x, 0)=x^{2}
$$

By construction the iteration formula as follows:

$$
\begin{aligned}
u_{n+1}(x, t)= & u_{n}(x, t)-\frac{1}{\Gamma(\alpha+1)} \\
& \times \int_{0}^{t}\left\{\frac{\partial^{\alpha} u_{n}}{\partial t^{\alpha}}+\frac{\partial u_{n}}{\partial x}-\frac{\partial^{2} u_{n}}{\partial x^{2}}\right. \\
& \left.-\frac{2 t^{2-\alpha}}{\Gamma(3-\alpha)}-2 x+2\right\}(d s)^{\alpha} .
\end{aligned}
$$




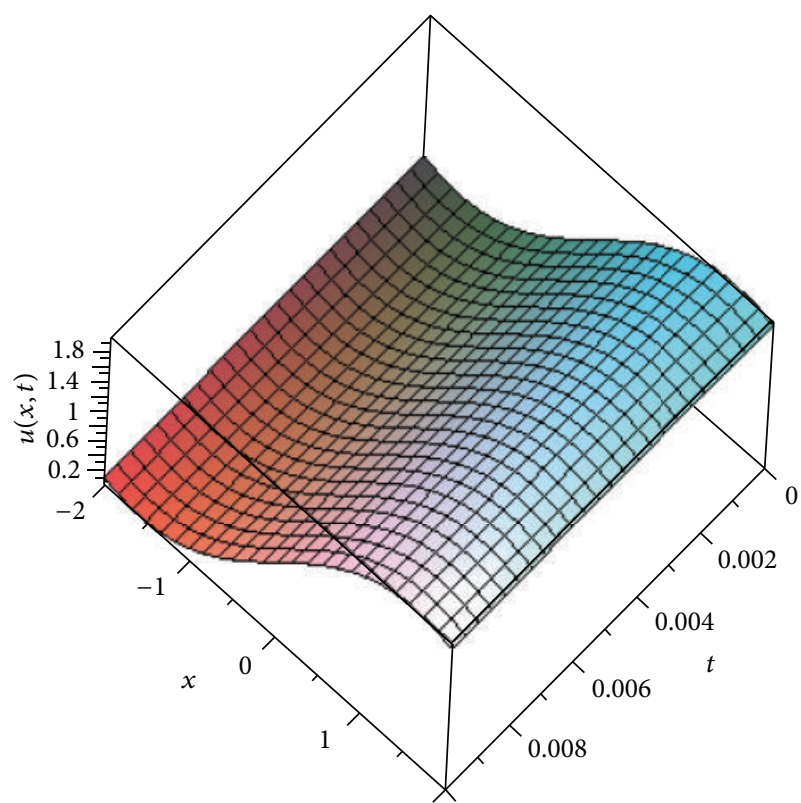

(a)

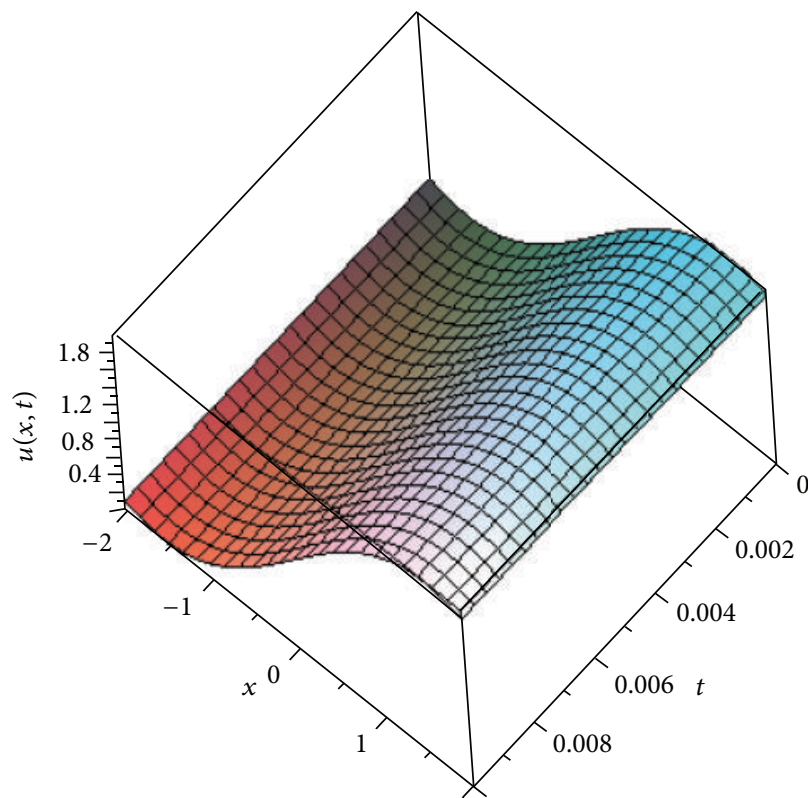

(b)

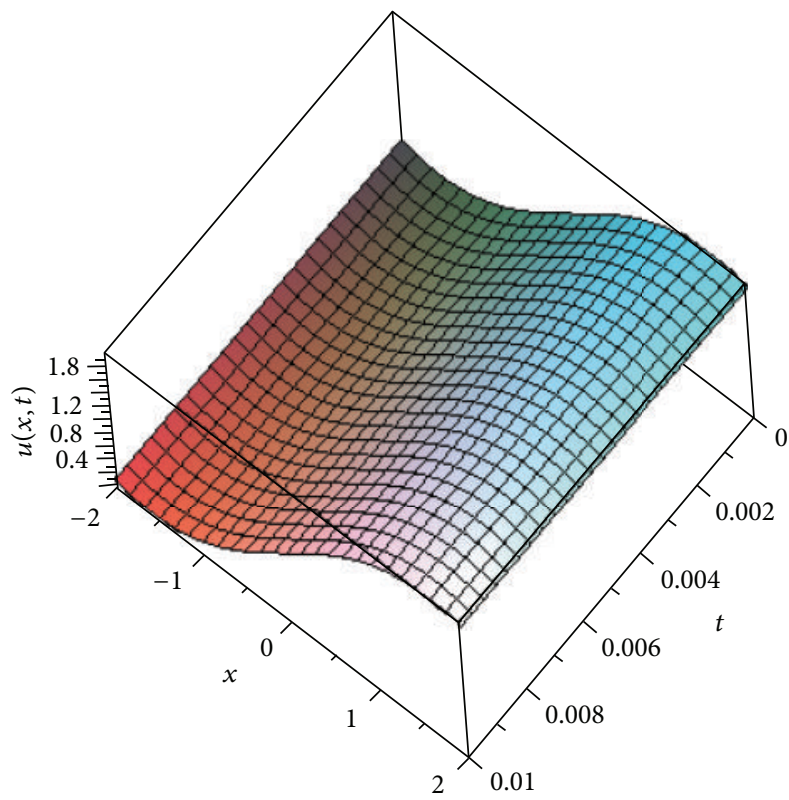

(c)

FIGURE 2: The surface shows the solution $u(x, t)$ for (27) with initial condition (28): (a) FVIM when $\alpha=1$, (b) HPM [7] when $\alpha=1$, and (c) exact solution.

Taking the initial value $u_{0}(x, t)=0$ we can derive the first approximate $u_{1}(x, t)$ as follows:

$$
\begin{aligned}
u_{1}(x, t)= & u_{0}(x, t)-\frac{1}{\Gamma(\alpha+1)} \\
& \times \int_{0}^{t}\left\{\frac{\partial^{\alpha} u_{0}}{\partial t^{\alpha}}+\frac{\partial u_{0}}{\partial x}-\frac{\partial^{2} u_{0}}{\partial x^{2}}\right. \\
& \left.\quad-\frac{t^{2-\alpha}}{\Gamma(3-\alpha)}-2 x+2\right\}(d s)^{\alpha} \\
= & x^{2}+t^{2},
\end{aligned}
$$

$$
\begin{aligned}
u_{2}(x, t)= & u_{1}(x, t)-\frac{1}{\Gamma(\alpha+1)} \\
& \times \int_{0}^{t}\left\{\frac{\partial^{\alpha} u_{1}}{\partial t^{\alpha}}+\frac{\partial u_{1}}{\partial x}-\frac{\partial^{2} u_{0}}{\partial x^{2}}\right. \\
& \left.\quad-\frac{t^{2-\alpha}}{\Gamma(3-\alpha)}-2 x+2\right\}(d s)^{\alpha} \\
= & x^{2}+t^{2} \\
\vdots & x^{2}+t^{2} .
\end{aligned}
$$




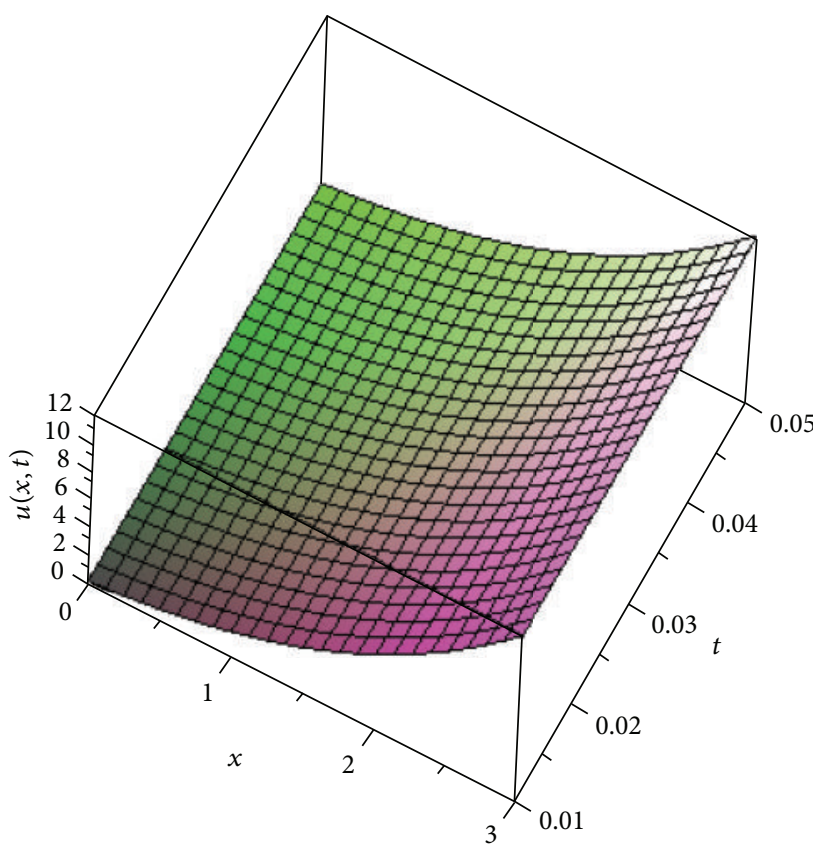

(a)

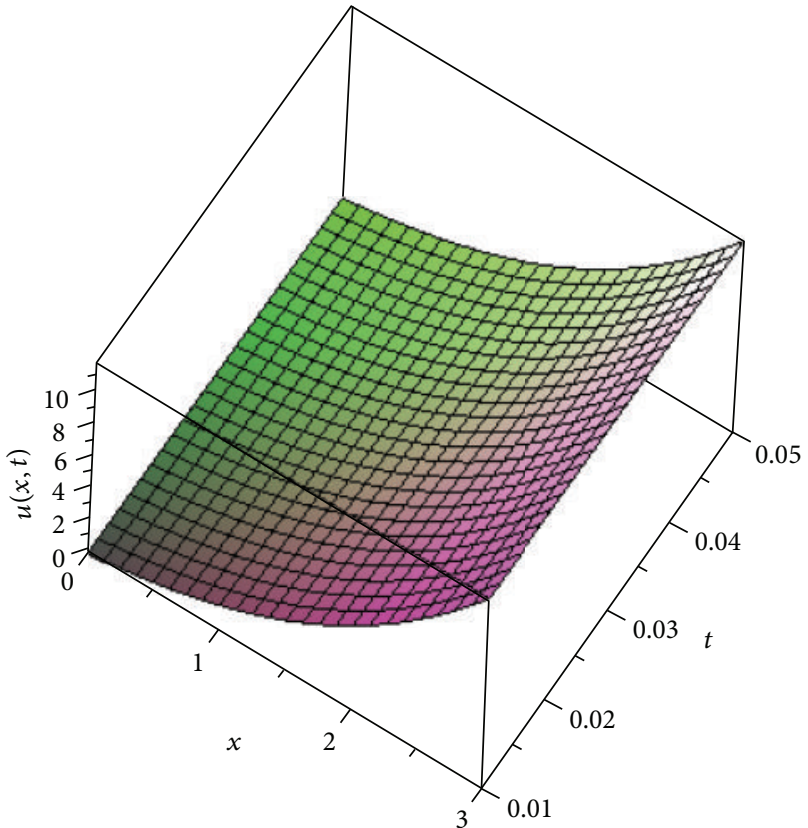

(b)

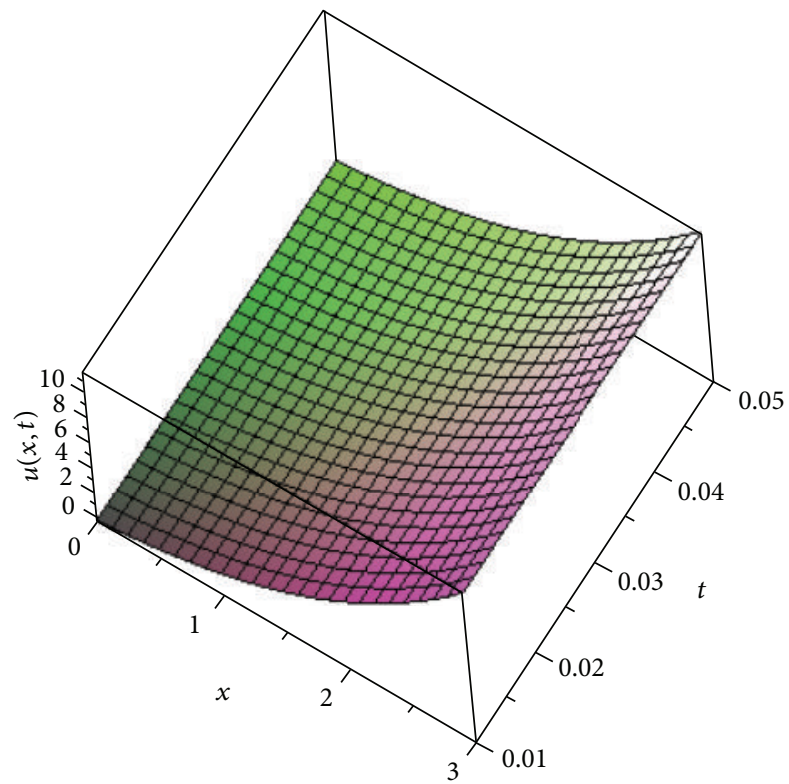

(c)

FIGURE 3: The surface shows the solution $u(x, t)$ for (36) with initial condition (37): (a) FVIM ( $\alpha=1)$, (b) HPM [10] ( $\alpha=1)$, and (c) FVIM $(\alpha=0.01)$.

So, the exact solution $u(x, t)=x^{2}+t^{2}$ follows immediately. The exact solution is obtained by using two iterations and this is dependent on proper selection of initial guess $u_{0}(x, t)$.

Example 10. We consider the following fractional BlackScholes option pricing equation [38] as follows:

$$
\frac{\partial^{\alpha} u}{\partial t^{\alpha}}=\frac{\partial^{2} u}{\partial x^{2}}+(k-1) \frac{\partial u}{\partial x}-k u, \quad 0<\alpha \leq 1,
$$

where $k$ is the risk-free interest rate subject to initial condition

$$
u(x, 0)=\max \left(e^{x}-1,0\right) .
$$

The exact solution for special case $\alpha=1$ is given by

$$
u(x, t)=\max \left(e^{x}-1,0\right) e^{-k t}+\max \left(e^{x}, 0\right)\left(1-e^{-k t}\right) .
$$


By construction the iteration formula as follows:

$$
\begin{aligned}
u_{n+1}(x, t)= & u_{n}(x, t)-\frac{1}{\Gamma(\alpha+1)} \\
& \times \int_{0}^{t}\left\{\frac{\partial^{\alpha} u_{n}}{\partial s^{\alpha}}-\frac{\partial^{2} u_{n}}{\partial x^{2}}\right. \\
& \left.+(k-1) \frac{\partial u_{n}}{\partial x}-k u_{n}\right\}(d s)^{\alpha} .
\end{aligned}
$$

Taking the initial value $u_{0}(x, t)=\max \left(e^{x}-1,0\right)$ we can derive the first approximate $u_{1}(x, t)$ as follows:

$$
\begin{aligned}
& u_{1}(x, t)=u_{0}(x, t)-\frac{1}{\Gamma(\alpha+1)} \\
& \times \int_{0}^{t}\left\{\frac{\partial^{\alpha} u_{0}}{\partial s^{\alpha}}-\frac{\partial^{2} u_{0}}{\partial x^{2}}+(k-1) \frac{\partial u_{0}}{\partial x}-k u_{0}\right\}(d s)^{\alpha} \\
& =\max \left(e^{x}-1,0\right)-\max \left(e^{x}, 0\right) \frac{\left(-k t^{\alpha}\right)}{\Gamma(\alpha+1)} \\
& +\max \left(e^{x}-1,0\right) \frac{\left(-k t^{\alpha}\right)}{\Gamma(\alpha+1)}, \\
& u_{2}(x, t)=u_{1}(x, t)-\frac{1}{\Gamma(\alpha+1)} \\
& \times \int_{0}^{t}\left\{\frac{\partial^{\alpha} u_{1}}{\partial s^{\alpha}}-\frac{\partial^{2} u_{1}}{\partial x^{2}}\right. \\
& \left.+(k-1) \frac{\partial u_{1}}{\partial x}-k u_{1}\right\}(d s)^{\alpha} \\
& =\max \left(e^{x}-1,0\right)-\max \left(e^{x}, 0\right) \\
& \times\left(\frac{\left(-k t^{\alpha}\right)}{\Gamma(\alpha+1)}+\frac{\left(-k t^{\alpha}\right)^{2}}{\Gamma(2 \alpha+1)}\right) \\
& +\max \left(e^{x}-1,0\right)\left(\frac{\left(-k t^{\alpha}\right)}{\Gamma(\alpha+1)}+\frac{\left(-k t^{\alpha}\right)^{2}}{\Gamma(2 \alpha+1)}\right) \\
& \vdots \\
& u_{3}(x, t)=u_{2}(x, t)-\frac{1}{\Gamma(\alpha+1)} \\
& \times \int_{0}^{t}\left\{\frac{\partial^{\alpha} u_{2}}{\partial s^{\alpha}}-\frac{\partial^{2} u_{2}}{\partial x^{2}}\right. \\
& \left.+(k-1) \frac{\partial u_{2}}{\partial x}-k u_{2}\right\}(d s)^{\alpha}
\end{aligned}
$$

$$
\begin{aligned}
= & \max \left(e^{x}-1,0\right)-\max \left(e^{x}, 0\right) \\
& \times\left(\frac{\left(-k t^{\alpha}\right)}{\Gamma(\alpha+1)}+\frac{\left(-k t^{\alpha}\right)^{2}}{\Gamma(2 \alpha+1)}+\frac{\left(-k t^{\alpha}\right)^{3}}{\Gamma(3 \alpha+1)}\right) \\
& +\max \left(e^{x}-1,0\right) \\
& \times\left(\frac{\left(-k t^{\alpha}\right)}{\Gamma(\alpha+1)}+\frac{\left(-k t^{\alpha}\right)^{2}}{\Gamma(2 \alpha+1)}+\frac{\left(-k t^{\alpha}\right)^{3}}{\Gamma(3 \alpha+1)}\right) \\
& \vdots \\
u_{n}(x, t)= & \max \left(e^{x}-1,0\right) E_{\alpha}\left(-k t^{\alpha}\right) \\
& +\max \left(e^{x}, 0\right)\left(1-E_{\alpha}\left(-k t^{\alpha}\right)\right),
\end{aligned}
$$

so that the solution $u(x ; t)$ of the problem is given by

$$
\begin{aligned}
u_{n}(x, t)= & \max \left(e^{x}-1,0\right) E_{\alpha}\left(-k t^{\alpha}\right) \\
& +\max \left(e^{x}, 0\right)\left(1-E_{\alpha}\left(-k t^{\alpha}\right)\right),
\end{aligned}
$$

where $E_{\alpha}(z)$ is Mittag-Leffler function in one parameter. Equation (41) represents the closed form solution of the fractional Black-Scholes equation (36). Now for the standard case $\alpha=1$, this series has the closed form of the solution $u(x ; t)=\max \left(e^{x}-1,0\right) e^{-k t}+\max \left(e^{x}, 0\right)\left(1-e^{-k t}\right)$, which is an exact solution of the given Black-Scholes equation (36) for $\alpha=1$.

In Figure 3 we have shown the surface of $u(x, t)$ corresponding to the value ( $\alpha=1$ for FVIM\&HPM and for FVIM $\alpha=0.01)$.

\section{Conclusion}

Variational iteration method has been known as a powerful method for solving many fractional equations such as partial differential equations, integrodifferential equations, and so many other equations. In this paper, based on the variational iteration method and modified Riemann-Liouville derivative, we have presented a general framework of fractional variational iteration method for analytical and numerical treatment of fractional partial differential equations in fluid mechanics and in financial models. All of the examples concluded that the fractional variational iteration method is powerful and efficient in finding analytical approximate solutions as well as numerical solutions. For example, the results of Examples 8 and 10 illustrate that the present method is in excellent agreement with those of HPM and exact solution, where the obtained solution is shown graphically. Further, in Example 9 we got the exact solution in two iterations. The basic idea described in this paper is expected to be further employed to solve other similar linear and nonlinear problems in fractional calculus. Maple has been used for presenting graph of solution in the present paper. 


\section{Acknowledgments}

The second author gratefully acknowledges that this research partially supported by Ministry of Higher Education (MOHE), Malaysia under the ERGS Grant 5527068.

\section{References}

[1] I. Podlubny, Fractional Differential Equations, vol. 198 of Mathematics in Science and Engineering, Academic Press, San Diego, Calif, USA, 1999.

[2] J. H. He, "Some applications of nonlinear fractional differential equations and their approximations," Bulletin of Science Technology \& Society, vol. 15, no. 2, pp. 86-90, 1999.

[3] J. H. He, "Nonlinear oscillation with fractional derivative and its applications," in Proceedings of the International Conference on Vibrating Engineering, pp. 288-291, Dalian, Chaina, 1998.

[4] A. Barone, F. Esposito, C. J. Magee, and A. C. Scott, "Theory and applications of the sine-gordon equation," La Rivista del Nuovo Cimento, vol. 1, no. 2, pp. 227-267, 1971.

[5] E. Yusufoglu, "The variational iteration method for studying the Klein-Gordon equation," Applied Mathematics Letters, vol. 21, no. 7, pp. 669-674, 2008.

[6] M. Khan, S. Hyder Ali, and H. Qi, "On accelerated flows of a viscoelastic fluid with the fractional Burgers' model," Nonlinear Analysis. Real World Applications. An International Multidisciplinary Journal, vol. 10, no. 4, pp. 2286-2296, 2009.

[7] A. K. Golmankhaneh, A. K. Golmankhaneh, and D. Baleanu, "On nonlinear fractional KleinGordon equation," Signal Processing, vol. 91, no. 3, pp. 446-451, 2011.

[8] M. Kurulay, "Solving the fractional nonlinear Klein-Gordon equation by means of the homotopy analysis method," Advances in Difference Equations, p. 2012187, 2012.

[9] E. Hesameddini and F. Fotros, "Solution for time-fractional coupled Klein-Gordon Schrodinger equation using decomposition method," International Mathematical Forum, vol. 7, no. 21-24, pp. 1047-1056, 2012.

[10] A. A. Elbeleze, A. Kiliçman, and B. M. Taib, "Homotopy perturbation method for fractional black-scholes european option pricing equations using Sumudu transform," Mathematical Problems in Engineering, vol. 2013, Article ID 524852, 7 pages, 2013.

[11] S. Kumar, A. Yildirim, Y. Khan, H. Jafari, K. Sayevand, and L. Wei, "Analytical solution of fractional Black-Scholes European option pricing equation by using Laplace transform," Journal of Fractional Calculus and Applications, vol. 2, no. 8, pp. 1-9, 2012.

[12] C. Xue, J. Nie, and W. Tan, "An exact solution of start-up flow for the fractional generalized Burgers' fluid in a porous half-space," Nonlinear Analysis. Theory, Methods \& Applications A: Theory and Methods, vol. 69, no. 7, pp. 2086-2094, 2008.

[13] M. Kurulay, "The approximate and exact solutions of the space and time-fractional Burggres equations," International Journal of Research and Reviews in Applied Sciences, vol. 3, no. 3, pp. 257-263, 2010.

[14] Z. Odibat and S. Momani, "The variational iteration method: an efficient scheme for handling fractional partial differential equations in fluid mechanics," Computers \& Mathematics with Applications, vol. 58, no. 11-12, pp. 2199-2208, 2009.

[15] D. D. Ganji and A. Sadighi, "Application of homotopyperturbation and variational iteration methods to nonlinear heat transfer and porous media equations," Journal of Computational and Applied Mathematics, vol. 207, no. 1, pp. 24-34, 2007.
[16] M. Rafei, D. D. Ganji, H. Daniali, and H. Pashaei, "The variational iteration method for nonlinear oscillators with discontinuities," Journal of Sound and Vibration, vol. 305, no. 4-5, pp. 614-620, 2007.

[17] D. D. Ganji, M. Jannatabadi, and E. Mohseni, "Application of He's variational iteration method to nonlinear JaulentMiodek equations and comparing it with ADM," Journal of Computational and Applied Mathematics, vol. 207, no. 1, pp. 3545, 2007.

[18] S. Momani and S. Abuasad, "Application of He's variational iteration method to Helmholtz equation," Chaos, Solitons \& Fractals, vol. 27, no. 5, pp. 1119-1123, 2006.

[19] J. H. He, "Variational iteration method for delay differential equations," Communications in Nonlinear Science and Numerical Simulation, vol. 2, no. 4, pp. 235-236, 1997.

[20] J. He, "Semi-inverse method of establishing generalized variational principles for fluid mechanics with emphasis on turbomachinery aerodynamics," International Journal of Turbo and Jet Engines, vol. 14, no. 1, pp. 23-28, 1997.

[21] J. H. He and X. H. Wu, "Variational iteration method: new development and applications," Computers \& Mathematics with Applications, vol. 54, no. 7-8, pp. 881-894, 2007.

[22] M. Inokuti, H. Sekine, and T. Mura, "General use of the Lagrange multiplier in non-linear mathematical physics," in Variational Method in the Mechanics of Solids, S. Nemat-Nasser, Ed., pp. 156-162, Pergamon Press, Oxford, UK, 1978.

[23] A. M. Wazwaz, "The variational iteration method for solving linear and nonlinear systems of PDEs," Computers \& Mathematics with Applications, vol. 54, no. 7-8, pp. 895-902, 2007.

[24] A. M. Wazwaz, "The variational iteration method: a reliable analytic tool for solving linear and nonlinear wave equations," Computers \& Mathematics with Applications, vol. 54, no. 7-8, pp. 926-932, 2007.

[25] A. M. Wazwaz, "The variational iteration method: a powerful scheme for handling linear and nonlinear diffusion equations," Computers \& Mathematics with Applications, vol. 54, no. 7-8, pp. 933-939, 2007.

[26] Z. Odibat, "Reliable approaches of variational iteration method for nonlinear operators," Mathematical and Computer Modelling, vol. 48, no. 1-2, pp. 222-231, 2008.

[27] E. Yusufoglu, "Variational iteration method for construction of some compact and noncompact structures of Klein-Gordon equations," International Journal of Nonlinear Sciences and Numerical Simulation, vol. 8, no. 2, pp. 153-158, 2007.

[28] J. Biazar and H. Ghazvini, "He's variational iteration method for solving hyperbolic differential equations," International Journal of Nonlinear Sciences and Numerical Simulation, vol. 8, no. 3, pp. 311-314, 2007.

[29] H. Ozer, "Application of the variational iteration method to the boundary value problems with jump discontinuities arising in solid mechanics," International Journal of Nonlinear Sciences and Numerical Simulation, vol. 8, no. 4, pp. 513-518, 2007.

[30] G. C. Wu and E. W. M. Lee, "Fractional variational iteration method and its application," Physics Letters A, vol. 374, no. 25, pp. 2506-2509, 2010.

[31] G. C. Wu, "New trends in the variational iteration method," Communications in Fractional Calculus, vol. 2, pp. 59-75, 2011.

[32] M. Merdan, "On the solutions fractional riccati differential equation with modified Riemann-Liouville derivative," International Journal of Differential Equations, vol. 2012, Article ID 346089, 17 pages, 2012. 
[33] G. Jumarie, "Stochastic differential equations with fractional Brownian motion input," International Journal of Systems Science, vol. 24, no. 6, pp. 1113-1131, 1993.

[34] G. Jumarie, "Laplace's transform of fractional order via the Mittag-Leffler function and modified Riemann-Liouville derivative," Applied Mathematics Letters, vol. 22, no. 11, pp. 16591664, 2009.

[35] G. Jumarie, "Table of some basic fractional calculus formulae derived from a modified Riemann-Liouville derivative for nondifferentiable functions," Applied Mathematics Letters, vol. 22, no. 3, pp. 378-385, 2009.

[36] A.-M. Wazwaz, "The variational iteration method for solving two forms of Blasius equation on a half-infinite domain," Applied Mathematics and Computation, vol. 188, no. 1, pp. 485491, 2007.

[37] F. Mainardi, "On the initial value problem for the fractional diffusion-wave equation," in Waves and Stability in Continuous Media, S. Rionero and T. Ruggeeri, Eds., pp. 246-251, World Scientific, Singapore, 1994.

[38] V. Gülkac, "The homotopy perturbation method for the BlackScholes equation," Journal of Statistical Computation and Simulation, vol. 80, no. 12, pp. 1349-1354, 2010. 


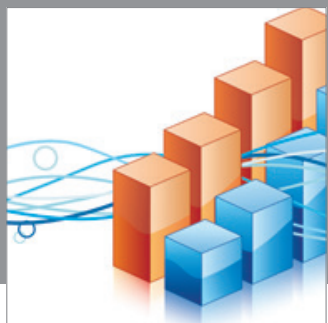

Advances in

Operations Research

mansans

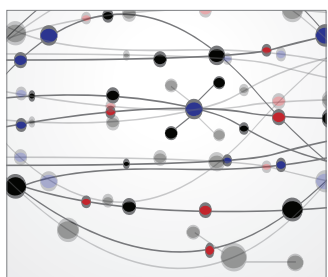

The Scientific World Journal
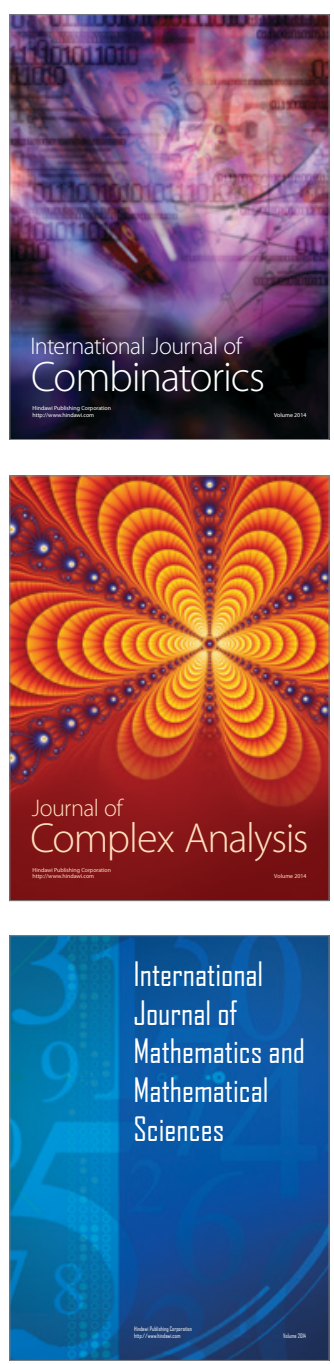
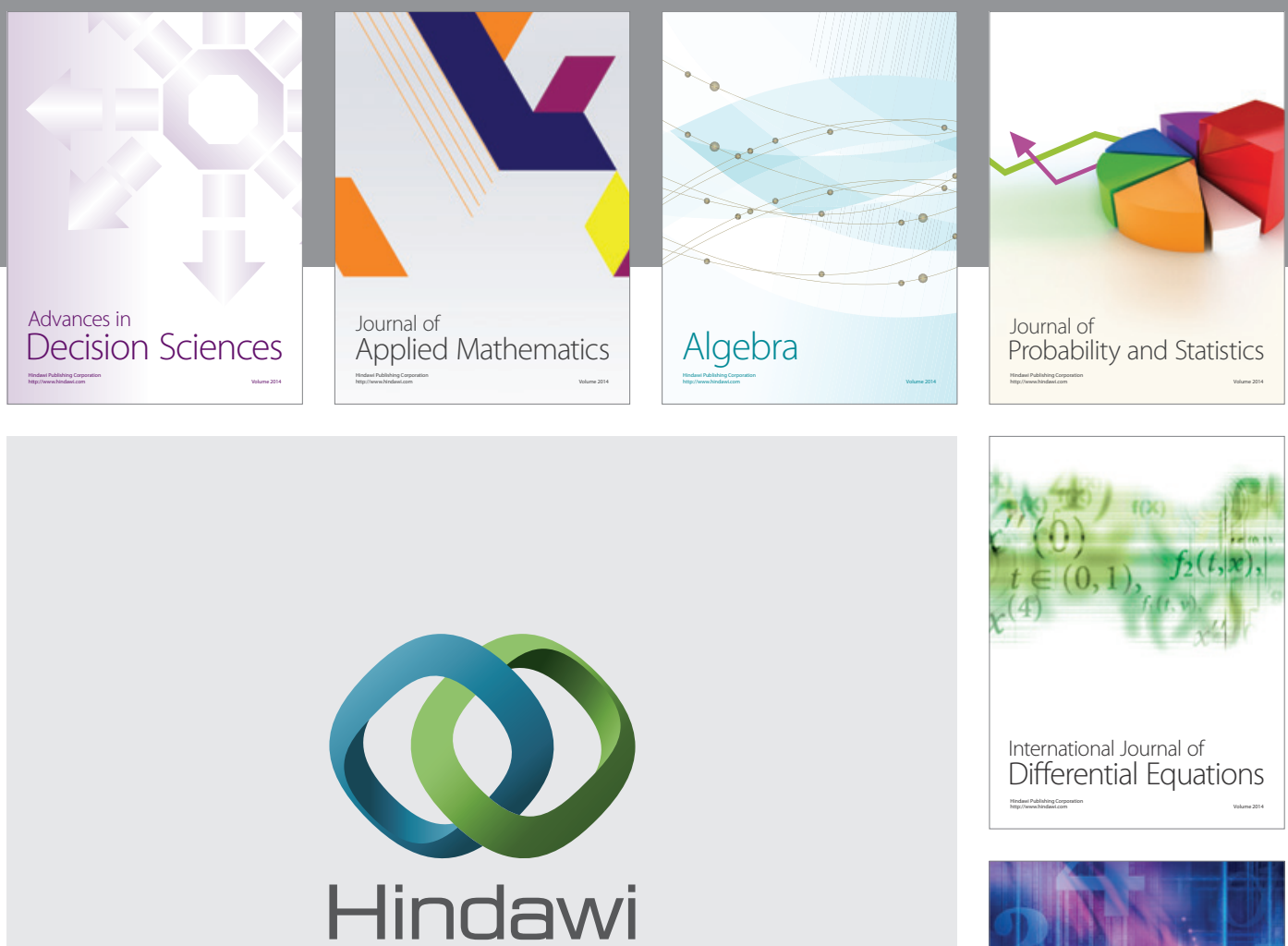

Submit your manuscripts at http://www.hindawi.com
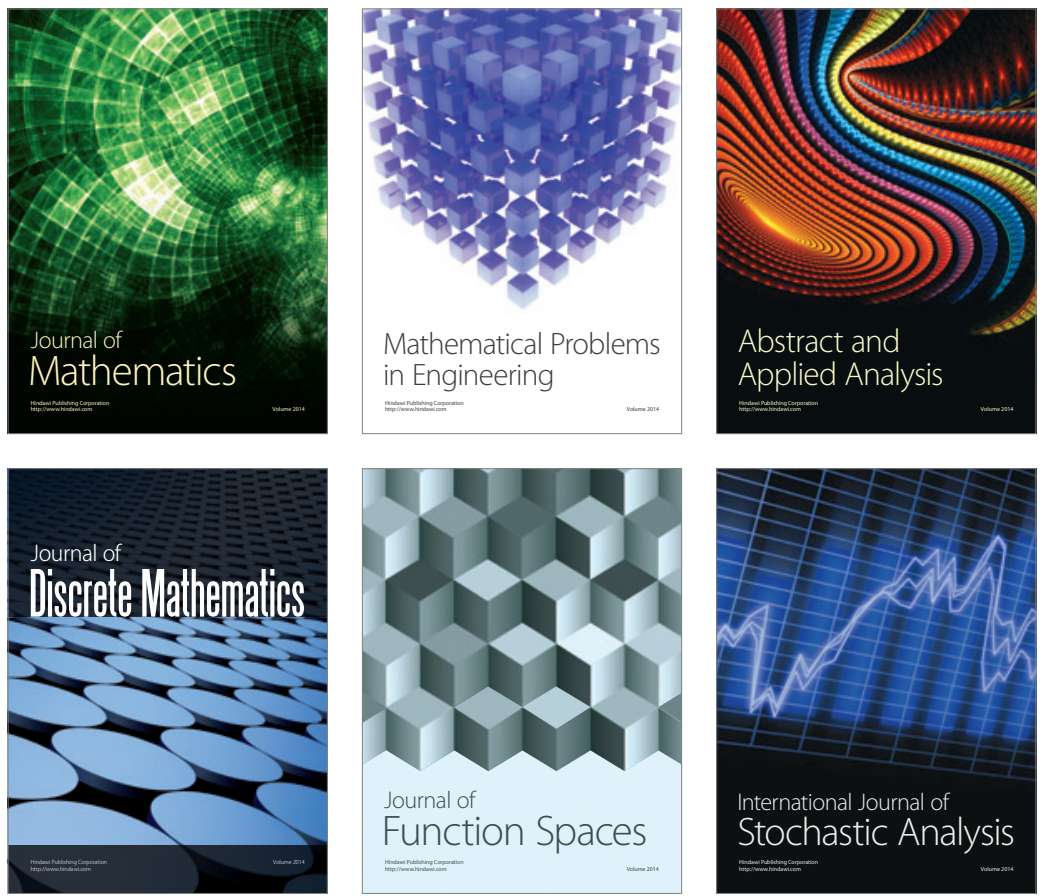

Journal of

Function Spaces

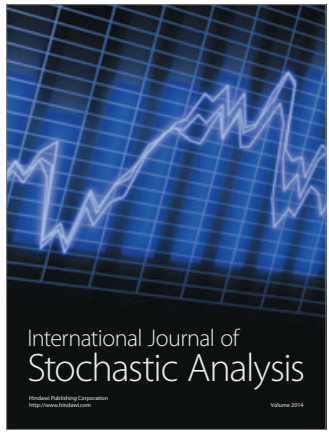

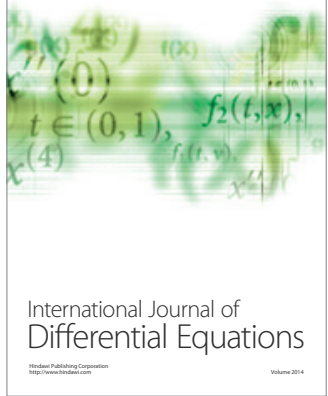
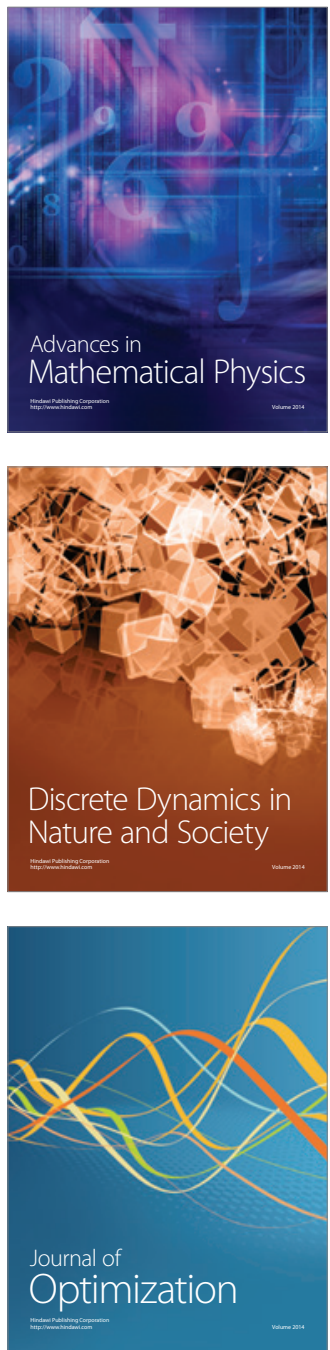